\title{
A high-fat, high-saturated fat diet decreases insulin sensitivity without changing intra-abdominal fat in weight-stable overweight and obese adults
}

\author{
Anize D. von Frankenberg ${ }^{1,2,6}$, Anna Marina ${ }^{1}$, Xiaoling Song ${ }^{3,4}$, Holly S. Callahan ${ }^{5}$, Mario \\ Kratz $^{1,3,4}$, and Kristina M. Utzschneider ${ }^{1,6}$ \\ ${ }^{1}$ Division of Metabolism, Endocrinology and Nutrition, Department of Medicine, University of \\ Washington, 1959 NE Pacific Street, Seattle, WA 98195, USA \\ ${ }^{2}$ Post-Graduate Endocrinology Program, School of Medicine, Universidade Federal do Rio \\ Grande do Sul, Porto Alegre, Brazil \\ ${ }^{3}$ Division of Public Health Sciences, Fred Hutchinson Cancer Research Center, 1100 Fairview \\ Ave N, Seattle, WA 98109, USA \\ ${ }^{4}$ Department of Epidemiology, University of Washington, 1959 NE Pacific Street, Seattle, WA \\ 98195, USA \\ ${ }^{5}$ School of Medicine, University of Washington, 1959 NE Pacific Street, Seattle, WA 98195, USA \\ ${ }^{6}$ Endocrinology, VA Puget Sound Health Care System, 1660 S Columbian Way (151), Seattle, WA \\ 98108, USA
}

\section{Abstract}

Purpose-We sought to determine the effects of dietary fat on insulin sensitivity and whether changes in insulin sensitivity were explained by changes in abdominal fat distribution or very lowdensity lipoprotein (VLDL) fatty acid composition.

Methods-Overweight/obese adults with normal glucose tolerance consumed a control diet (35\% fat $/ 12 \%$ saturated fat $/ 47 \%$ carbohydrate) for 10 days, followed by a 4-week low-fat diet (LFD, $n=10: 20 \%$ fat $/ 8 \%$ saturated fat $/ 62 \%$ carbohydrate) or high-fat diet (HFD, $n=10: 55 \%$ fat $/ 25 \%$ saturated fat $/ 27 \%$ carbohydrate). All foods and their eucaloric energy content were provided. Insulin sensitivity was measured by labeled hyperinsulinemic-euglycemic clamps, abdominal fat distribution by MRI, and fasting VLDL fatty acids by gas chromatography.

Results-The rate of glucose disposal (Rd) during low-and high-dose insulin decreased on the HFD but remained unchanged on the LFD (Rd-low: LFD: $0.12 \pm 0.11$ vs. HFD: $-0.37 \pm 0.15$ $\mathrm{mmol} / \mathrm{min}$, mean $\pm \mathrm{SE}, p<0.01$; Rdhigh: LFD: $0.11 \pm 0.37 \mathrm{vs.} \mathrm{HFD:}-0.71 \pm 0.26 \mathrm{mmol} / \mathrm{min}, p$

Correspondence to: Anize D. von Frankenberg.

Electronic supplementary material The online version of this article (doi:10.1007/s00394-015-1108-6) contains supplementary material, which is available to authorized users.

ClinicalTrials.gov Identifier NCT00930371.

Compliance with ethical standard

Conflict of interest The authors declare that they have no conflict of interest. 
$=0.08)$. Hepatic insulin sensitivity did not change. Changes in subcutaneous fat were positively associated with changes in insulin sensitivity on the LFD $(r=0.78, p<0.01)$ with a trend on the HFD $(r=0.60, p=0.07)$, whereas there was no association with intra-abdominal fat. The LFD led to an increase in VLDL palmitic (16:0), stearic (18:0), and palmitoleic $(16: 1 \mathrm{n} 7 \mathrm{c})$ acids, while no changes were observed on the HFD. Changes in VLDL n-6 docosapentaenoic acid (22:5n6) were strongly associated with changes in insulin sensitivity on both diets (LFD: $r=-0.77 ; p<0.01$; HFD: $r=-0.71 ; p=0.02$ ).

Conclusions-A diet very high in fat and saturated fat adversely affects insulin sensitivity and thereby might contribute to the development of type 2 diabetes.

\section{Keywords}

Dietary fat; Saturated fat; High-fat diet; Insulin sensitivity

\section{Introduction}

Type 2 diabetes is reaching epidemic proportions worldwide with the World Health Organization estimating that worldwide $9 \%$ of adults older than age 18 have diabetes with the vast majority having type 2 diabetes [1]. Elevated hepatic glucose production, impaired insulin secretion, and insulin resistance, a typical complication of obesity, are major factors underlying the pathogenesis of type 2 diabetes [2]. Weight loss induced by lifestyle measures including diet and physical activity has been shown to decrease the risk of developing diabetes, with a $16 \%$ reduction in risk for every kilogram of weight loss [3]. Insulin sensitivity is improved by hypocaloric dietary interventions irrespective of whether they are low or high in fat content [4-6], but this effect may be attributed to weight loss itself rather than diet composition. Thus, to determine the effects of dietary macronutrient composition on insulin sensitivity, it is important that they be tested in the absence of weight change.

Medium- to long-term diet intervention studies have examined whether isocaloric high-fat diets (HFDs) modify insulin sensitivity. Two studies that compared a HFD (50-55\% of calories as fat) versus a low-fat diet (LFD) (20-25\% of calories as fat) demonstrated no difference in insulin sensitivity measured by clamp, in healthy adults after 3 and 2 weeks, respectively [7, 8]. We also observed no significant change in fasting insulin concentrations or the Matsuda index measure of insulin sensitivity after 4 weeks on a high-fat/highsaturated fat diet (43\% calories from fat $/ 24 \%$ saturated fat) in weight-stable older subjects [9]. Additionally, after 11 days on isocaloric low-fat, intermediate-fat, or high-fat diets ( 0 , 41 , and $83 \%$ of fat, respectively), insulin sensitivity did not differ between the high- and low-fat diets [10]. These data are at odds with the preconception that HFD leads to insulin resistance.

In contrast, a LFD with less than $10 \%$ of energy from saturated fat improved insulin sensitivity after 24 weeks [11]. We also observed an improvement in the Matsuda index after 4 weeks on a low-fat/low-saturated fat/low-glycemic index diet [9]. LFDs are by default higher in carbohydrate content if protein content is kept stable. Carbohydrate content alone may modify insulin sensitivity with one study showing a significant increase in this 
parameter after eating a very high-carbohydrate diet (85\%) [12]. Diets high in carbohydrates also provide substrate to stimulate de novo lipogenesis in the liver [13, 14] and result in production of fatty acids such as palmitic (16:0) and stearic (18:0) acids that have been shown to be related to decreased insulin sensitivity [15]. However, increases in palmitoleate acid (16:1n7c) after a LFD may promote insulin sensitivity in white adipose tissue [15]. Thus, dietary effects on fatty acid composition may influence effects on insulin sensitivity and warrant investigation.

In addition to fatty acids, body fat composition and ectopic fat storage are thought to be important factors in regulating glucose metabolism [16]. Potential mediators from adipose tissue include free fatty acids, inflammatory cytokines and adipokines such as leptin and adiponectin [17]. Studies have associated increased intra-abdominal fat (IAF) [18], but not subcutaneous fat (SQF), with decreased insulin sensitivity [19], and fat accumulation in the liver has been associated with insulin resistance [20]. IAF is an important source of nonesterified fatty acids (NEFAs) in the portal circulation [21], draining them directly to the liver and thereby altering hepatic glucose and lipid metabolism $[22,23]$ and contributing to insulin resistance [24]. Recently, the impact of dietary fat on body fat deposition has been explored. In cross-sectional studies, diets high in saturated fat are associated with increased total body and trunk fat deposition compared to diets low in fat [25]. Two studies that examined the effect of altering diet fat quality but kept total dietary fat intake constant found decreases in SQF with diets high in polyunsaturated fatty acid (PUFA) [26, 27]. To our knowledge, only two studies have investigated the effect of a high-fat, high-saturated fat versus a low-fat, low-saturated fat diet on body fat distribution in weight-stable subjects. Neither found a significant effect on IAF or SQF $[9,20]$. However, we observed an increase in abdominal SQF after 4 weeks on a high-fat/high-saturated fat diet despite weight stability [28]. Whether such changes in abdominal fat distribution contribute to effects of dietary fat content on insulin sensitivity is unclear.

In this study, we sought to determine the effect of diets containing either low or high amounts of fat and saturated fat on determinants of glucose tolerance, specifically insulin sensitivity, both in insulin's ability to suppress endogenous glucose production (EGP) and to promote glucose uptake, and insulin secretion in weight-stable overweight/ obese subjects. We hypothesized that a diet high in fat would decrease insulin sensitivity, while a diet low in fat would improve insulin sensitivity. Fiber intake was standardized across the different intervention arms so that this would not be a confounding factor. Since few previous studies have looked into mechanisms by which HFDs affect insulin sensitivity, we further investigated possible mediators of diet-induced changes in insulin sensitivity by examining changes in abdominal fat distribution, adipokines, and VLDL fatty acid analysis. VLDL fatty acids were measured as the primary goal of the study was to evaluate the effect of dietary fat content on liver fat and its relationship to insulin sensitivity. We have previously published that the HFD led to increases in SQF [28], but examine here whether such changes in body fat distribution contribute to changes in insulin sensitivity. 


\section{Research design and methods}

\section{Study design}

The study was a prospective, random order, crossover, controlled dietary feeding study. Details on the study design have been previously published [28]. Briefly, subjects completed a 10-day control diet followed by 4 weeks on either a LFD or HFD. All food was provided to the participants during the control, HFD, and LFD periods. The seven subjects who completed both the HFD and LFD underwent a 6-week washout period during which subjects ate $a d l i b$ at home and no food was provided. The control diet was then repeated prior to the second intervention diet.

A portion of the data have been published previously [28] but are reproduced here for ease of reference.

Subjects-The study enrolled men and women between the ages of 18-55 years with BMI $>27 \mathrm{~kg} / \mathrm{m}^{2}$ and normal glucose tolerance based on fasting $(<5.5 \mathrm{mmol} / \mathrm{L}$ or $<100 \mathrm{mg} / \mathrm{dl})$ and 2-h glucose $(<7.8 \mathrm{mmol} / \mathrm{L}$ or $<140 \mathrm{mg} / \mathrm{dl})$ levels after a standard $75 \mathrm{~g}$ oral glucose tolerance test. Exclusion criteria included tobacco use, significant medical illness, reported alcohol consumption $>2$ alcoholic drinks/ day, alanine aminotransferase $>40 \mathrm{U} / \mathrm{L}$, serum creatinine $>132.6 \mu \mathrm{mol} / \mathrm{L}(>1.5 \mathrm{mg} / \mathrm{dl})$ in men and $>123.8 \mu \mathrm{mol} / \mathrm{L}(>1.4 \mathrm{mg} / \mathrm{dl})$ in women, hematocrit $<33 \%$, fasting triglycerides $>3.4 \mathrm{mmol} / \mathrm{L}$ ( $>300 \mathrm{mg} / \mathrm{dl}$ ), fasting LDL cholesterol $>5.2 \mathrm{mmol} / \mathrm{L}$ (>200 mg/dl), food allergies/intolerances, contraindications to magnetic resonance imaging (MRI), and use of any medications affecting inflammation, insulin sensitivity, or liver fat. All subjects gave written informed consent. The study was approved by the Institutional Review Boards of the Veterans Affairs Puget Sound Health Care System and the University of Washington in accordance with ethical standards on human experimentation.

\section{Dietary intervention}

Menus were designed by a research nutritionist using Pro-Nutra (VioCare, Inc., Princeton, $\mathrm{NJ})$ to contain the following: control: $35 \%$ energy from fat (12\% saturated fat), $47 \%$ energy from carbohydrate, and $18 \%$ energy from protein; LFD: $20 \%$ energy from fat (8\% saturated fat), $62 \%$ energy from carbohydrate, and $18 \%$ energy from protein; and HFD: $55 \%$ fat ( $25 \%$ saturated fat), $27 \%$ energy from carbohydrate, and $18 \%$ energy from protein. Caloric needs were estimated using the average of the Mifflin-St. Jeor [29] and Harris-Benedict [30] equations, adjusted for physical activity. Major sources of fats in all three diets included butter and high oleic safflower oil. Soluble fiber (inulin) was added to the HFD to standardize fiber content across diets. Because fructose was limited on the HFD due to the low carbohydrate content, fructose was limited in all diets to $<30 \mathrm{~g} /$ day based on a $2000 \mathrm{kcal} / \mathrm{day}$. The mean fructose intake was higher on the LFD and lower on the HFD. The composition of the diets is described in Table 1.

Subjects picked up their food from the metabolic kitchen and were weighed twice weekly. Caloric intake was adjusted to achieve weight stability. Subjects were instructed to maintain regular physical activity and to eat all of the food provided, not to eat any non-study food, 
and to report any deviations from the diet. To determine compliance, subjects recorded all food consumed each day using a checklist which was returned to the nutritionist. All foods that were not consumed were returned to the Nutrition Research Kitchen and weighed to determine the actual energy intake and composition of consumed foods.

\section{Study procedures}

Study procedures were performed at the end of the control diet and at the end of the LFD or HFD. Subjects were told to fast for at least $10 \mathrm{~h}$ before undergoing study procedures.

Intravenous glucose tolerance test-An intravenous glucose tolerance test (IVGTT) was performed to assess the acute insulin response to glucose (AIRg) and glucose tolerance. An intravenous line was established in an antecubital vein, and the arm was wrapped in a heating pad to "arterialize" the blood. A bolus of glucose $\left(11.4 \mathrm{~g} / \mathrm{m}^{2}\right)$ was injected over $60 \mathrm{~s}$, and blood samples were drawn at $-10,-5,-1,2,3,4,5,6,8,10,12,14,16,19,22,25$, and 30 min relative to the start of the glucose injection.

Hyperinsulinemic-euglycemic clamp-A two-step hyperinsulinemic-euglycemic clamp with 6,6-2d glucose isotope label was performed on the following day to estimate EGP and insulin sensitivity. After obtaining a baseline blood sample, a primed $\left(200 \mathrm{mg} / \mathrm{m}^{2}\right.$ $\times$ glucose $/ 100$ over $5 \mathrm{~min}$ ), continuous $\left(2 \mathrm{mg} / \mathrm{m}^{2} / \mathrm{min}\right.$ ) infusion of $6,6-2 \mathrm{~d}$ glucose was started and continued throughout the clamp procedure. Following a $3-\mathrm{h}$ basal equilibration period, a 3-h low-dose insulin infusion $\left(20 \mathrm{mU} / \mathrm{m}^{2} / \mathrm{min}\right)$ followed by a 2-h primed, continuous highdose insulin infusion $\left(160 \mathrm{mU} / \mathrm{m}^{2} / \mathrm{min} \times 5 \mathrm{~min}\right.$ then $\left.80 \mathrm{mU} / \mathrm{m}^{2} / \mathrm{min}\right)$ was performed. Blood glucose was measured every 5 min using an iStat machine, and a variable rate infusion of $20 \%$ dextrose enriched with $2 \% 6,6-2 \mathrm{~d}$ glucose was titrated to maintain the blood glucose concentration at $90 \mathrm{mg} / \mathrm{dl}$. Samples were drawn for glucose and insulin every $30 \mathrm{~min}$ throughout the clamp. Samples for glucose, insulin, and 6,6-2d glucose were drawn every 15 min during the final half hour of the basal, low-dose, and high-dose insulin infusion periods. Samples for NEFAs were drawn into tubes containing the lipolysis inhibitor tetrahydrolipstatin (orlistat) at $-30,-15,-1,10,20,30$, and 60 min relative to the start of the low-dose insulin infusion and placed immediately on ice. NEFA samples were processed within $30 \mathrm{~min}$, and the plasma flash-frozen.

\section{Fat distribution}

Total fat and lean mass were determined on the first control diet by dual-energy X-Ray absorptiometry using the QDR ${ }^{\circledR}$ 4500A bone densitometer system (Hologic, Inc. Bedford, MA).

Abdominal fat distribution (IAF and SQF) and liver fat were measured using MRI/MRS abdominal images as previously described [9]. The inter- and intra-scan coefficients of variation (CVs) were 4.9 and $2.4 \%$ for IAF and 6.2 and $3.1 \%$ for SQF, respectively. MRS was used to quantify hepatic triglyceride using a Philips Achieva 3 Tesla, version 2.5.3.0 (Philips, Andover, MA) whole body scanner. The inter- and intra-scan CVs for liver fat were 18.6 and $1.2 \%$, respectively. 
Assays-The following assays were performed: glucose by glucose oxidase; insulin by automated electrochemiluminescence immunoassay (Cobas e601, Indianapolis, IN); and adiponectin and leptin by radioimmunoassay (Millipore, Billerica, MA). Intra- and interassay CVs (\%) for the adiponectin assay were 6.21 and 9.25 and for the leptin assay were 3.7 and 5.2, respectively. VLDL fatty acids were isolated by gradient ultracentrifugation. Fatty acid methyl ester samples were prepared by direct trans-esterification using the method of Lepage [31] and separated using gas chromatography (Agilent 5890 gas chromatograph with FID detector and ChemSation software; Supelco fused silica 100-m capillary column SP-2560; initial $160{ }^{\circ} \mathrm{C}$ for $16 \mathrm{~min}$, ramp $3.0^{\circ} \mathrm{C} / \mathrm{min}$ to $240{ }^{\circ} \mathrm{C}$, hold for $15 \mathrm{~min}$ ) (Agilent Technology, Santa Clara, CA). The CV (\%) ranged from 0.7 to 13.1 depending on the type of VLDL fatty acid. Levels of 6,6-2d glucose were measured by mass spectrometry as previously described [32].

\section{Calculations}

IVGTT data-The AIRg was calculated as the AUC insulin response above basal from 0 to $10 \mathrm{~min}$. AIRg was adjusted for insulin sensitivity measured by the clamp method to estimate beta-cell function. The glucose disappearance constant $(\mathrm{Kg})$, a measure of intravenous glucose tolerance, was calculated as the slope of the natural log of glucose from 10 to 30 $\min$.

Clamp data-Isotopic steady-state concentrations were achieved during the final $30 \mathrm{~min}$ of the basal and low- and high-dose insulin periods of the clamp. The rates of glucose appearance ( $\mathrm{Ra}$ ) and disappearance $(\mathrm{Rd})$ were calculated based on steady-state equations modified to include the use of a labeled dextrose infusion [33]. EGP was determined in the basal state and at the end of the low-dose glucose infusion. Hepatic insulin sensitivity was determined both by the percent suppression of EGP from basal at the end of the low-dose insulin infusion and as the hepatic insulin resistance index (HIR index: basal EGP $\times$ fasting plasma insulin).

\section{Statistical analysis}

Data were expressed as mean \pm standard error (SE) for normally distributed data or median (interquartile range) for non-normally distributed data. Generalized estimating equation (GEE) analysis was performed to determine the effect of diet type on the change in each outcome variable (intervention diet—respective control diet), adjusted for diet order and type. The GEE method focuses on average changes in response over time and the impact of covariates on these changes. Unlike RM-ANOVA, GEE does not require the outcome variable to have a normal distribution and permits use of all available data (even if the subject did not complete all study phases) in an unbalanced design, leading to more efficient effect estimates. Individuals with missing data are considered a random subset of the sample. This feature benefits crossover studies in which missing data occurred and/or data are skewed due to a small sample size [34].

The significance of the associations between changes in insulin sensitivity and changes in abdominal fat distribution, adipokines, and VLDL fatty acid composition were tested using nonparametric Spearman's correlation coefficient. A $p<0.05$ was considered significant. 
Statistical analyses were performed using SPSS software (version 9.0, SPSS Inc., Chicago, IL).

\section{Results}

\section{Subject characteristics}

This was a prospective, crossover study where a total of 13 subjects $(10 \mathrm{M} / 3 \mathrm{~F}: 3$ African Americans, 1 Asian, and 9 Caucasians; age $36 \pm 2.9$ years; BMI $33.6 \pm 1.3 \mathrm{~kg} / \mathrm{m}^{2}$; fasting glucose $5.0 \pm 0.1 \mathrm{mmol} / \mathrm{L} ; 2$-h glucose $5.3 \pm 0.3 \mathrm{mmol} / \mathrm{L}$ ) completed diets and procedures. Seven participants completed both intervention diets (control + LFD and control + HFD, $n=$ 7), and six participants completed only one of the diet interventions (control + LFD, $n=3$ ) or (control $+\mathrm{HFD}, n=3$ ). All available data were included in the analysis. All participants except two reported consuming all food provided [28]. Removing these two subjects from all analyses did not change the results.

\section{Response to the LFD}

Compared to the control diet, body weight did not change. The Kg increased significantly after the LFD (Table 2), demonstrating improved glucose tolerance, despite no significant change in AIRg. Glucose and insulin levels were well matched during the clamp (Fig. 1a, c). There was no significant change in insulin sensitivity as measured by the rate of glucose disposal (Rd) during the low- and high-level insulin infusions (Fig. 1e), HIR index, basal EGP, or insulin-mediated suppression of EGP on the LFD compared to the control diet (Table 2). Additionally, during the clamp there was no difference in the ability of low-dose insulin to suppress free fatty acids (Fig. 1g). As previously published [28], liver fat decreased significantly during the LFD, but there were no significant changes in fasting glucose and insulin, IAF, SQF or adipokines (Table 2).

Changes in the percent fatty acid composition of VLDL are shown in Table 3 . The proportion of stearic acid (18:0) increased significantly, whereas palmitic acid (16:0) showed a trend to increase during the LFD. Among mono-unsaturated fatty acids (MUFA), palmitoleic acid (16:1n7c) showed an increase during the LFD. Finally, the most abundant PUFA, linoleic acid (18:2n6), decreased significantly during the LFD.

\section{Response to the HFD}

Compared to the control diet, there were no significant changes in body weight, $\mathrm{AIRg}, \mathrm{Kg}$, HIR index, EGP, or EGP suppression during the HFD (Table 2). Glucose and insulin levels were well matched during the clamp (Fig. 1b, d). There was a significant decrease in Rd but no change in the ability of low-dose insulin to suppress free fatty acids (Fig. 1f, h). There was no significant change in IAF or liver fat; however, there was a significant increase in SQF with the HFD. All other metabolic parameters did not change (Table 2).

There was no change in the percent fatty acid composition of VLDL during the HFD (Table 3). 


\section{Comparison of the LFD and HFD}

Changes from control were compared between the LFD and HFD adjusted for diet order and type. There was a significant difference in the change in Rd-low (LFD: $0.12 \pm 0.11$ vs. HFD: $-0.37 \pm 0.15 \mathrm{mmol} / \mathrm{min}$, mean $\pm \mathrm{SE}, p<0.01$ ) with a trend for Rd-high (LFD: $0.11 \pm 0.37$ vs. HFD: $-0.71 \pm 0.26 \mathrm{mmol} / \mathrm{min}, p=0.08)$. Kg was significantly improved on the LFD compared to the HFD (LFD: $0.26 \pm 0.12$ vs. HFD: $-0.36 \pm 0.18 \% / \mathrm{min}, p<0.01$ ). The increase in SQF on the HFD was significant compared to the change on the LFD (LFD: 9.3 \pm 42.9 vs. HFD: $156.4 \pm 42.3 \mathrm{~cm}^{3}, p=0.02$ ) (Table 2). There were no significant differences between changes on the LFD versus the HFD in the other metabolic variables.

Correlates of changes in insulin sensitivity-After the LFD, changes in SQF were positively associated with changes in Rd-low (Supplemental Figure 1A: $r=0.78 ; p<0.01$ ) and with changes in Rd-high $(r=0.83 ; p<0.01)$. After the HFD, increases in SQF also tended to be positively associated with changes in Rd-low (Supplemental Figure 1B: $r=$ $0.60 ; p=0.07)$ but not with changes in Rd-high $(r=-0.52 ; p=0.13)$. There was no association between changes in Rd-low or Rd-high and IAF on either the LFD (Rd-low: $r=$ $-0.10 ; p=0.78$; Rd-high: $r=0.20 ; p=0.58$ ) or HFD (Rd-low: $r=-0.26 ; p=0.47$; Rd-high: $r=0.39 ; p=0.21$ ) (Supplemental Figure 1C,1D). There were no significant associations between changes in Rd-low or Rd-high and changes in the SQF/IAF ratio, liver fat, or adipokines (data not shown) after either diet.

Among VLDL fatty acids, changes in VLDL n-6 docosapentaenoic acid (22:5n6) were strongly negatively associated with changes in Rd-low after both diets (LFD: $r=-0.77 ; p<$ 0.01; HFD: $r=-0.71 ; p=0.02$ ) (Supplemental Figure 2). An increase in palmitic acid (16:0) was associated with an increase in hepatic insulin resistance after the LFD $(r=0.79 ; p=$ $0.01)$ but not after the HFD $(r=0.07 ; p=0.86)$. There were no significant associations between changes in any of the other VLDL fatty acids and changes in Rd, HIR, or percent suppression of EGP.

After either LFD or HFD, there were no correlations between changes in HIR index and changes in SQF, IAF, or liver fat. Also, after both LFD and HFD there were no associations between changes in EGP suppression and changes in SQF, IAF, or liver fat.

Correlates of changes in glucose tolerance-After the LFD, changes in Kg were not associated with changes in AIRg, Rd-low, or Rd-high. After the HFD, changes in Kg were positively associated with changes in AIRg $(r=0.82 ; p<0.01)$ but not with changes in Rdlow or Rd-high.

\section{Discussion}

Four weeks on a diet very high in fat and saturated fat significantly decreased insulin sensitivity in overweight/obese subjects despite the absence of weight gain. However, a diet low in fat and saturated fat did not improve insulin sensitivity. The decrease in insulin sensitivity on the HFD could not be explained by changes in IAF, liver fat, or adipokines. However, positive correlations between changes in SQF and insulin sensitivity were 
observed. Intriguingly, changes in VLDL n-6 docosapentaenoic acid (22:5n6) were strongly negatively correlated with changes in insulin sensitivity on both the HFDs and LFDs.

We explored potential mechanisms related to the decrease in insulin sensitivity on the HFD. The strongest association we observed was a negative association between changes in insulin sensitivity and changes in VLDL n-6 docosapentaenoic acid (22:5n6). This association was observed on both the HFD and the LFD. This fatty acid is the end-product of n-6 PUFA desaturation and elongation. Although such a correlation does not imply a causal role, the strength of the correlation despite small changes is intriguing and further study into the role of n-6 docosapentaenoic acid (22:5n6) in metabolic processes is warranted. Unfortunately, data regarding the relative amounts of this fatty acid are lacking in the literature.

The VLDL fatty acid profile is strongly correlated with the fatty acid profile within the liver as assessed by liver biopsies [35]. On a balanced diet (30\% as fat and $55 \%$ as carbohydrate), approximately $15 \%$ of the hepatic tri-glyceride is derived from the diet [35]. We anticipated that increasing the dietary contribution of saturated fatty acids would lead to increased saturated fatty acids within the liver and contribute to hepatic insulin resistance. However, we observed changes in Rd, reflecting mainly uptake of glucose into muscle, and no changes in measures of hepatic insulin sensitivity.

Other possible mechanisms whereby increased dietary fat intake decreases insulin sensitivity include decreases in cell membrane responsiveness to insulin action through decreases in binding affinity [36]. Others have reported an exaggerated synthesis of ceramides from a HFD enriched with saturated fatty acid (i.e., palmitic acid 16:0), which might also induce insulin resistance [37]. While others have proposed it is mediated by increases in inflammatory cytokines [38], we did not observe any changes in inflammatory markers [28] and no associations between changes in these markers and insulin sensitivity despite the very high-saturated fat intake in our study (unpublished observations). This would argue against inflammation as a major underlying mechanism.

In contrast to the findings on the HFD, no improvement in insulin sensitivity was observed on the LFD. One possible explanation is that changes in insulin sensitivity on the HFD were driven by the high-saturated fat content rather than total dietary fat intake. If this were the case, the lack of change in insulin sensitivity on the LFD may have been due in part to the relatively small change in saturated fat ( $11.9 \%$ control to $7.7 \%$ LFD). In contrast, the difference in saturated fat content between the control diet and the HFD (11.6-23.7\%) was quite large. In a previous study, a high-saturated fat diet (17\% of energy from saturated fat) reduced insulin sensitivity by $12.5 \%$ after 3 months of intervention in healthy subjects compared to baseline [39]. Our study was designed to compare a HFD with a LFD and was not specifically designed to determine the effect of dietary saturated fat per se. Other isoenergetic feeding studies have compared high PUFA versus high saturated fat or high MUFA versus saturated fat on insulin sensitivity [26, 39-42]. A single liquid meal high in PUFA improved post-prandial insulin sensitivity as compared to a high-fat, high-saturated fat meal [41]. Additionally, after $24 \mathrm{~h}$, high-saturated fat ingestion decreased insulin sensitivity compared to both control and high-PUFA interventions [40]. Longer-term studies showed increases in insulin sensitivity after 6 and 12 weeks on a diet containing large amounts of 
MUFA compared to a high-carbohydrate or a high-saturated fat diet [39, 42], and after 5 weeks on a diet high in PUFA versus saturated fat [26].

Another possible explanation for the finding that insulin sensitivity did not improve in the LFD compared to the control diet may be that the effect of dietary fat intake on insulin sensitivity is not linear, or that there may be a threshold effect that reduces insulin sensitivity only at very high fat intake levels. It is possible that the LFD, which by default contained higher carbohydrates, stimulated deleterious metabolic pathways that counterbalanced beneficial effects leading to no net benefit. One such pathway is hepatic de novo lipogenesis, which is known to be driven by high carbohydrate intake. The increase we observed in the proportion of VLDL palmitic (16:0) and stearic (18:0) acids, both saturated fatty acids, on the LFD likely reflects an increase in de novo lipogenesis [43]. There is evidence that stearic acid (18:0) in the diet or as free fatty acid induces insulin resistance [44]. Moreover, in a recent cohort study, palmitic (16:0) and stearic (18:0) acids, measured in plasma phospholipids, were positively associated with incident type 2 diabetes [45]. In contrast, the increase in VLDL palmitoleate (16:1n7c) observed on the LFD might mediate insulinsensitizing effects, in part due to suppressing pro-inflammatory gene expression in white adipose tissue which has been observed in mice [46]; however, there are conflicting data in humans $[47,48]$. One observational study found no difference in palmitoleate acid (16:1n7c) content, measured in both plasma and VLDL, in insulin-sensitive or insulin-resistant obese subjects, which suggests that there is no association between palmitoleate acid (16:1n7c) availability and insulin resistance [47]. In a prospective study, decreasing content of free fatty acid palmitoleate acid (16:1n7c) was associated with improvement in insulin sensitivity after 1 year of a lifestyle intervention; however, this effect was not independent of lifestyle changes [48]. Therefore, any potential benefit in insulin sensitivity associated with an increase in palmitoleate acid $(16: 1 \mathrm{n} 7 \mathrm{c})$ could have been attenuated by increases in palmitic (16:0) and stearic (18:0) acids resulting in no net benefit.

Despite the lack of effect on insulin sensitivity, the LFD did result in an improvement in glucose tolerance. However, there were no changes in beta-cell function or insulin sensitivity after the LFD. Moreover, we did not find associations between improvement in glucose tolerance with changes in AIRg or Rd after the LFD to explain this finding.

The strengths of our study include the controlled diet intervention, weight stability, measurement of abdominal fat distribution, and the use of labeled clamps to measure insulin sensitivity. There are some limitations to the present study. While the HFD was designed to be high in saturated fat, our study was designed to compare the effects of a low- versus highfat diet and not specifically designed to compare saturated fat versus other types of fat. Thus, conclusions about the effect of the high saturated fat content versus total fat content cannot be drawn. Second, because of the small sample size, small effects of the LFD on insulin sensitivity and effects of both diets on secondary outcome variables might have been missed. Third, we studied subjects with normal glucose tolerance and normal liver enzymes. Thus, our findings reflect relatively healthy overweight/obese adults who may be more able to quickly adapt to changes in dietary lipid intake. The results therefore cannot necessarily be extrapolated to individuals with impaired glucose metabolism or diabetes. Additionally, by design the HFD contained very high fat and saturated fat, which is not typical of a diet 
consumed by free-living individuals. Total fat and saturated fats contribute about 33 and $10 \%$ of energy to the North American diet, respectively [49]. However, even though this amount of fat is not typically consumed by the general population, our findings do demonstrate that dietary fat can impact insulin sensitivity. Finally, the complexity of dietary composition manipulation does not permit matching of all nutrients. While maintaining protein intake stable, as dietary fat content decreases, carbohydrate content increases and vice versa. The very low carbohydrate content on the HFD also prevented matching of fructose content, although this was limited in all diets. The higher fructose content in the LFD is unlikely to have impacted our results as the average difference between the control and LFD diet was only $12 \mathrm{~g}$ per day and both were relatively low in fructose. We did match fiber intake as this has been shown to affect glucose metabolism [50].

\section{Conclusions}

Based on a significant decrease in insulin sensitivity after a diet very high in fat and saturated fat, we conclude that such a diet may be detrimental for glucose homeostasis and could contribute to the development of type 2 diabetes. While the low-fat, low-saturated fat diet did improve intravenous glucose tolerance $(\mathrm{Kg})$, we failed to observe any improvement in insulin sensitivity. We hypothesize that this could be related to counterbalancing effects of higher carbohydrate intake driving de novo synthesis of detrimental fatty acids.

\section{Supplementary Material}

Refer to Web version on PubMed Central for supplementary material.

\section{Acknowledgments}

We are grateful to the study participants for their contribution and time. This study was supported by funding and resources from the Department of Veteran Affairs, Diabetes Research Center (P30DK017047), the Institute for Translational Health Sciences (UL1TR000423), the Nutrition Obesity Research Center (P30 DK035816), the Diagnostic Imaging Sciences Center at the University of Washington, and the Brazilian Federal Agency for Support and Evaluation of Graduate Education (CAPES) (AF).

\section{Abbreviations}

AIRg Acute insulin response to glucose

EGP Endogenous glucose production

GEE Generalized estimating equation

Kg Glucose disappearance constant

HIR index Hepatic insulin resistance index

HFD High-fat diet

IAF Intra-abdominal fat

IVGTT Intravenous glucose tolerance test

LFD Low-fat diet 


$\begin{array}{ll}\text { MRI } & \text { Magnetic resonance imaging } \\ \text { NEFAs } & \text { Non-esterified fatty acids } \\ \text { PUFA } & \text { Polyunsaturated fatty acid } \\ \text { Ra } & \text { Rate of glucose appearance } \\ \text { Rd } & \text { Rate of glucose disposal } \\ \text { SQF } & \text { Subcutaneous fat } \\ \text { VLDL } & \text { Very low-density lipoprotein }\end{array}$

\section{References}

1. WHO. Global status report on noncommunicable diseases 2014. Geneva: WHO; 2014.

2. Kahn SE, Hull RL, Utzschneider KM. Mechanisms linking obesity to insulin resistance and type 2 diabetes. Nature. 2006; 444(7121):840-846. [PubMed: 17167471]

3. Hamman RF, Wing RR, Edelstein SL, Lachin JM, Bray GA, Delahanty L, Hoskin M, Kriska AM, Mayer-Davis EJ, Pi-Sunyer X, Regensteiner J, Venditti B, Wylie-Rosett J. Effect of weight loss with lifestyle intervention on risk of diabetes. Diabetes Care. 2006; 29(9):2102-2107. [PubMed: 16936160]

4. Shai I, Schwarzfuchs D, Henkin Y, Shahar DR, Witkow S, Greenberg I, Golan R, Fraser D, Bolotin A, Vardi H, Tangi-Rozental O, Zuk-Ramot R, Sarusi B, Brickner D, Schwartz Z, Sheiner E, Marko R, Katorza E, Thiery J, Fiedler GM, Bluher M, Stumvoll M, Stampfer MJ. Dietary Intervention Randomized Controlled Trial G. Weight loss with a low-carbohydrate, Mediterranean, or low-fat diet. N Engl J Med. 2008; 359(3):229-241. [PubMed: 18635428]

5. Guldbrand H, Dizdar B, Bunjaku B, Lindstrom T, Bachrach-Lindstrom M, Fredrikson M, Ostgren CJ, Nystrom FH. In type 2 diabetes, randomisation to advice to follow a low-carbohydrate diet transiently improves glycaemic control compared with advice to follow a low-fat diet producing a similar weight loss. Diabetologia. 2012; 55(8):2118-2127. [PubMed: 22562179]

6. Guldbrand H, Lindstrom T, Dizdar B, Bunjaku B, Ostgren CJ, Nystrom FH, Bachrach-Lindstrom M. Randomization to a low-carbohydrate diet advice improves health related quality of life compared with a low-fat diet at similar weight-loss in type 2 diabetes mellitus. Diabetes Res Clin Pract. 2014

7. van Herpen NA, Schrauwen-Hinderling VB, Schaart G, Mensink RP, Schrauwen P. Three weeks on a high-fat diet increases intrahepatic lipid accumulation and decreases metabolic flexibility in healthy overweight men. J Clin Endocrinol Metab. 2011; 96(4):E691-E695. [PubMed: 21252252]

8. Eckel RH, Hernandez TL, Bell ML, Weil KM, Shepard TY, Grunwald GK, Sharp TA, Francis CC, Hill JO. Carbohydrate balance predicts weight and fat gain in adults. Am J Clin Nutr. 2006; 83(4): 803-808. [PubMed: 16600931]

9. Utzschneider KM, Bayer-Carter JL, Arbuckle MD, Tidwell JM, Richards TL, Craft S. Beneficial effect of a weight-stable, low-fat/low-saturated fat/low-glycaemic index diet to reduce liver fat in older subjects. Br J Nutr. 2013; 109(6):1096-1104. [PubMed: 22849970]

10. Bisschop PH, de Metz J, Ackermans MT, Endert E, Pijl H, Kuipers F, Meijer AJ, Sauerwein HP, Romijn JA. Dietary fat content alters insulin-mediated glucose metabolism in healthy men. Am J Clin Nutr. 2001; 73(3):554-559. [PubMed: 11237931]

11. Jebb SA, Lovegrove JA, Griffin BA, Frost GS, Moore CS, Chatfield MD, Bluck LJ, Williams CM, Sanders TA, Group RS. Effect of changing the amount and type of fat and carbohydrate on insulin sensitivity and cardiovascular risk: the RISCK (Reading, Imperial, Surrey, Cambridge, and Kings) trial. Am J Clin Nutr. 2010; 92(4):748-758. [PubMed: 20739418]

12. Chen M, Bergman RN, Porte D Jr. Insulin resistance and beta-cell dysfunction in aging: the importance of dietary carbohydrate. J Clin Endocrinol Metab. 1988; 67(5):951-957. [PubMed: 3053750] 
13. Sjostrom L. Carbohydrate-stimulated fatty acid synthesis de novo in human adipose tissue of different cellular types. Acta Med Scand. 1973; 194(5):387-404. [PubMed: 4757218]

14. Angel A, Bray GA. Synthesis of fatty acids and cholesterol by liver, adipose tissue and intestinal mucosa from obese and control patients. Eur J Clin Invest. 1979; 9(5):355-362. [PubMed: 118029]

15. Hudgins LC, Hellerstein M, Seidman C, Neese R, Diakun J, Hirsch J. Human fatty acid synthesis is stimulated by a eucaloric low fat, high carbohydrate diet. J Clin Investig. 1996; 97(9):20812091. [PubMed: 8621798]

16. Jazet IM, Pijl H, Meinders AE. Adipose tissue as an endocrine organ: impact on insulin resistance. Neth J Med. 2003; 61(6):194-212. [PubMed: 12948164]

17. Mlinar B, Marc J. New insights into adipose tissue dysfunction in insulin resistance. Clin Chem Lab Med. 2011; 49(12):1925-1935. [PubMed: 21892913]

18. Cnop M, Havel PJ, Utzschneider KM, Carr DB, Sinha MK, Boyko EJ, Retzlaff BM, Knopp RH, Brunzell JD, Kahn SE. Relationship of adiponectin to body fat distribution, insulin sensitivity and plasma lipoproteins: evidence for independent roles of age and sex. Diabetologia. 2003; 46(4): 459-469. [PubMed: 12687327]

19. Hsieh CJ, Wang PW, Chen TY. The relationship between regional abdominal fat distribution and both insulin resistance and subclinical chronic inflammation in non-diabetic adults. Diazbetol Metab Syndr. 2014; 6(1):49.

20. Westerbacka J, Lammi K, Hakkinen AM, Rissanen A, Salminen I, Aro A, Yki-Jarvinen H. Dietary fat content modifies liver fat in overweight nondiabetic subjects. J Clin Endocrinol Metab. 2005; 90(5):2804-2809. [PubMed: 15741262]

21. Arner P. Differences in lipolysis between human subcutaneous and omental adipose tissues. Ann Med. 1995; 27(4):435-438. [PubMed: 8519504]

22. Fisher EA, Ginsberg HN. Complexity in the secretory pathway: the assembly and secretion of apolipoprotein B-containing lipoproteins. J Biol Chem. 2002; 277(20):17377-17380. [PubMed: 12006608]

23. Rebrin K, Steil GM, Getty L, Bergman RN. Free fatty acid as a link in the regulation of hepatic glucose output by peripheral insulin. Diabetes. 1995; 44(9):1038-1045. [PubMed: 7657026]

24. Boden G. Role of fatty acids in the pathogenesis of insulin resistance and NIDDM. Diabetes. 1997; 46(1):3-10. [PubMed: 8971073]

25. Neville MM, Geppert J, Min Y, Grimble G, Crawford MA, Ghebremeskel K. Dietary fat intake, body composition and blood lipids of university men and women. Nutr Health. 2012; 21(3):173185. [PubMed: 23533205]

26. Summers LK, Fielding BA, Bradshaw HA, Ilic V, Beysen C, Clark ML, Moore NR, Frayn KN. Substituting dietary saturated fat with polyunsaturated fat changes abdominal fat distribution and improves insulin sensitivity. Diabetologia. 2002; 45(3):369-377. [PubMed: 11914742]

27. Tapsell LC, Batterham MJ, Teuss G, Tan SY, Dalton S, Quick CJ, Gillen LJ, Charlton KE. Longterm effects of increased dietary polyunsaturated fat from walnuts on metabolic parameters in type II diabetes. Eur J Clin Nutr. 2009; 63(8):1008-1015. [PubMed: 19352378]

28. Marina A, van Frankenberg AD, Suvag S, Callahan HS, Kratz M, Richards TL, Utzschneider KM. Effects of dietary fat and saturated fat content on liver fat and markers of oxidative stress in overweight/obese men and women under weight-stable conditions. Nutrients. 2014; 6(11):46784690. [PubMed: 25353663]

29. Mifflin MD, St Jeor ST, Hill LA, Scott BJ, Daugherty SA, Koh YO. A new predictive equation for resting energy expenditure in healthy individuals. Am J Clin Nutr. 1990; 51(2):241-247. [PubMed: 2305711]

30. Harris, JABF. A biometric study of basal metabolism in man. Washington, DC: Carnegie Institution of Washington; 1919.

31. Lepage GRC. Direct transesterification of all classes of lipids in a one-step reaction. J Lipid Res. 1999; 27:114-120.

32. Yarasheski KE, Cade WT, Overton ET, Mondy KE, Hubert S, Laciny E, Bopp C, Lassa-Claxton S, Reeds DN. Exercise training augments the peripheral insulin-sensitizing effects of pioglitazone in HIV-infected adults with insulin resistance and central adiposity. Am J Physiol Endocrinol Metab. 2011; 300(1):E243-E251. [PubMed: 20959530] 
33. Wolfe, RR. Radioactive and stable isotope tracers in biomedicine. Nerw York: Wiley; 1992.

34. Ma Y, Mazumdar M, Memtsoudis SG. Beyond repeated-measures analysis of variance: advanced statistical methods for the analysis of longitudinal data in anesthesia research. Reg Anesth Pain Med. 2012; 37(1):99-105. [PubMed: 22189576]

35. Donnelly KL, Smith CI, Schwarzenberg SJ, Jessurun J, Boldt MD, Parks EJ. Sources of fatty acids stored in liver and secreted via lipoproteins in patients with nonalcoholic fatty liver disease. J Clin Investig. 2005; 115(5):1343-1351. [PubMed: 15864352]

36. Manco M, Calvani M, Mingrone G. Effects of dietary fatty acids on insulin sensitivity and secretion. Diabetes Obes Metab. 2004; 6(6):402-413. [PubMed: 15479216]

37. Eissing L, Scherer T, Todter K, Knippschild U, Greve JW, Buurman WA, Pinnschmidt HO, Rensen SS, Wolf AM, Bartelt A, Heeren J, Buettner C, Scheja L. De novo lipogenesis in human fat and liver is linked to ChREBP-beta and metabolic health. Nat Commun. 2013; 4:1528. [PubMed: 23443556]

38. Kennedy A, Martinez K, Chuang CC, LaPoint K, McIntosh M. Saturated fatty acid-mediated inflammation and insulin resistance in adipose tissue: mechanisms of action and implications. $\mathbf{J}$ Nutr. 2009; 139(1):1-4. [PubMed: 19056664]

39. Vessby B, Uusitupa M, Hermansen K, Riccardi G, Rivellese AA, Tapsell LC, Nalsen C, Berglund L, Louheranta A, Rasmussen BM, Calvert GD, Maffetone A, Pedersen E, Gustafsson IB, Storlien LH, Study K. Substituting dietary saturated for monounsaturated fat impairs insulin sensitivity in healthy men and women: the KANWU Study. Diabetologia. 2001; 44(3):312-319. [PubMed: 11317662]

40. Xiao C, Giacca A, Carpentier A, Lewis GF. Differential effects of monounsaturated, polyunsaturated and saturated fat ingestion on glucose-stimulated insulin secretion, sensitivity and clearance in overweight and obese, non-diabetic humans. Diabetologia. 2006; 49(6):1371-1379. [PubMed: 16596361]

41. Jans A, Konings E, Goossens GH, Bouwman FG, Moors CC, Boekschoten MV, Afman LA, Muller M, Mariman EC, Blaak EE. PUFAs acutely affect triacylglycerol-derived skeletal muscle fatty acid uptake and increase postprandial insulin sensitivity. Am J Clin Nutr. 2012; 95(4):825-836. [PubMed: 22338035]

42. Gadgil MD, Appel LJ, Yeung E, Anderson CA, Sacks FM, Miller ER3rd. The effects of carbohydrate, unsaturated fat, and protein intake on measures of insulin sensitivity: results from the OmniHeart trial. Diabetes Care. 2013; 36(5):1132-1137. [PubMed: 23223345]

43. Aarsland A, Wolfe RR. Hepatic secretion of VLDL fatty acids during stimulated lipogenesis in men. J Lipid Res. 1998; 39(6):1280-1286. [PubMed: 9643360]

44. van den Berg SA, Guigas B, Bijland S, Ouwens M, Voshol PJ, Frants RR, Havekes LM, Romijn JA, van Dijk KW. High levels of dietary stearate promote adiposity and deteriorate hepatic insulin sensitivity. Nutr Metab. 2010; 7:24.

45. Forouhi NG, Koulman A, Sharp SJ, Imamura F, Kroger J, Schulze MB, Crowe FL, Huerta JM, Guevara M, Beulens JW, van Woudenbergh GJ, Wang L, Summerhill K, Griffin JL, Feskens EJ, Amiano P, Boeing H, Clavel-Chapelon F, Dartois L, Fagherazzi G, Franks PW, Gonzalez C, Jakobsen MU, Kaaks R, Key TJ, Khaw KT, Kuhn T, Mattiello A, Nilsson PM, Overvad K, Pala V, Palli D, Quiros JR, Rolandsson O, Roswall N, Sacerdote C, Sanchez MJ, Slimani N, Spijkerman AM, Tjonneland A, Tormo MJ, Tumino R, van der ADL, van der Schouw YT, Langenberg C, Riboli E, Wareham NJ. Differences in the prospective association between individual plasma phospholipid saturated fatty acids and incident type 2 diabetes: the EPIC-InterAct case-cohort study. Lancet Diabetes Endocrinol. 2014; 2(10):810-818. [PubMed: 25107467]

46. Yang ZH, Miyahara H, Hatanaka A. Chronic administration of palmitoleic acid reduces insulin resistance and hepatic lipid accumulation in KK-Ay Mice with genetic type 2 diabetes. Lipids Health Dis. 2011; 10:120. [PubMed: 21774832]

47. Fabbrini E, Magkos F, Su X, Abumrad NA, Nejedly N, Coughlin CC, Okunade AL, Patterson BW, Klein S. Insulin sensitivity is not associated with palmitoleate availability in obese humans. J Lipid Res. 2011; 52(4):808-812. [PubMed: 21266364]

48. Corpeleijn E, Feskens EJ, Jansen EH, Mensink M, Saris WH, de Bruin TW, Blaak EE. Improvements in glucose tolerance and insulin sensitivity after lifestyle intervention are related to 
changes in serum fatty acid profile and desaturase activities: the SLIM study. Diabetologia. 2006; 49(10):2392-2401. [PubMed: 16896932]

49. Wright JD, Wang CY, Kennedy-Stephenson J, Ervin RB. Dietary intake of ten key nutrients for public health, United States: 1999-2000. Adv Data. 2003; 334:1-4.

50. Silva FM, Kramer CK, de Almeida JC, Steemburgo T, Gross JL, Azevedo MJ. Fiber intake and glycemic control in patients with type 2 diabetes mellitus: a systematic review with meta-analysis of randomized controlled trials. Nutr Rev. 2013; 71(12):790-801. [PubMed: 24180564] 

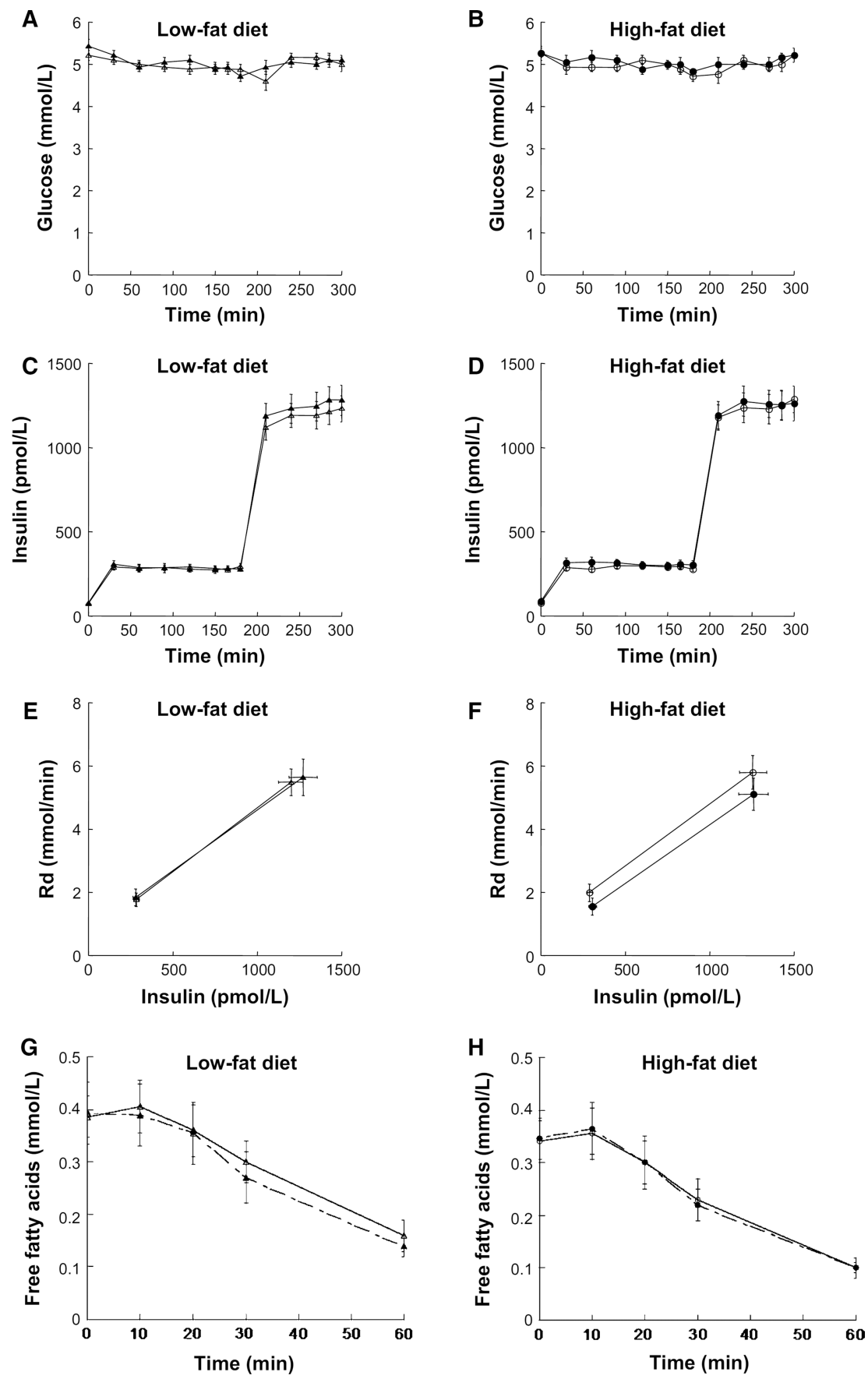

Fig. 1.

Glucose (a, b) and insulin (c, d) levels were well matched during the clamps. The Rd did not change on the LFD (e) but decreased significantly on the HFD (Rd-low $p=0.03$, Rd-high $p$ $=0.05)(\mathbf{f})$. There was no difference in free fatty acid suppression after the LFD $(\mathbf{g})$ and the HFD (h). Symbols: control prior to LFD open triangle and solid line; LFD solid triangle and dashed line; and control prior to HFD open circle and solid line; HFD solid circle and dashed line. Mean \pm SEM, $n=10$ for each diet 


\section{Table 1}

\section{Diet composition}

\begin{tabular}{lcccc}
\hline & Control LFD & LFD & Control HFD & HFD \\
\hline Daily energy (kcal) & $3284.0 \pm 125.0$ & $3321.0 \pm 150.0$ & $3140.0 \pm 120.0$ & $3208.0 \pm 92.0$ \\
Fat (\% of total energy) & $35.8 \pm 0.6$ & $20.2 \pm 0.0^{1}$ & $35.2 \pm 0.0$ & $54.8 \pm 0.1^{2,3}$ \\
Saturated fat (\% of total energy) & $11.9 \pm 0.4$ & $7.7 \pm 0.0^{1}$ & $11.6 \pm 0.0$ & $23.7 \pm 0.1^{2,3}$ \\
MUFA (\% of total energy) & $16.7 \pm 0.1$ & $7.7 \pm 0.0^{1}$ & $16.6 \pm 0.0$ & $22.2 \pm 0.1^{2,3}$ \\
PUFA (\% of total energy) & $4.7 \pm 0.0$ & $3.0 \pm 0.0^{1}$ & $4.7 \pm 0.0$ & $5.2 \pm 0.0^{2,3}$ \\
n-6 PUFA (\% of total energy) & $2.7 \pm 0.1$ & $2.0 \pm 0.0^{1}$ & $2.8 \pm 0.0$ & $3.8 \pm 0.1^{2,3}$ \\
n-3 PUFA (\% of total energy) & $0.1 \pm 0.0$ & $0.2 \pm 0.0^{1}$ & $0.1 \pm 0.0$ & $0.3 \pm 0.0^{2,3}$ \\
Cholesterol (mg/day) & $378.0 \pm 10.0$ & $492.0 \pm 21.01$ & $352.0 \pm 11.0$ & $506.0 \pm 17.0^{2}$ \\
Trans-fat (\% of total energy) & $0.8 \pm 0.0$ & $0.5 \pm 0.0^{1}$ & $0.8 \pm 0.0$ & $1.4 \pm 0.0^{2,3}$ \\
Protein (\% of total energy) & $17.9 \pm 0.0$ & $18.1 \pm 0.0$ & $17.8 \pm 0.0$ & $17.8 \pm 0.0^{3}$ \\
Carbohydrate (\% of total energy) & $46.4 \pm 0.6$ & $61.7 \pm 0.0$ & $46.9 \pm 0.0$ & $27.4 \pm 0.1^{2,3}$ \\
Total fiber (g/day) & $47.2 \pm 2.0$ & $46.1 \pm 2.1$ & $45.8 \pm 1.8$ & $39.8 \pm 1.3^{2,3}$ \\
Fructose (g/day) & $34.1 \pm 1.8$ & $46.1 \pm 2.11$ & $33.1 \pm 1.4$ & $10.0 \pm 0.3^{2,3}$ \\
\hline
\end{tabular}

Data for the study diet composition is inclusive of all subjects who completed the control and corresponding LFD or HFD ( $n=10$ for each). Mean diet composition data for the subset of subjects $(n=7)$ who completed both diet protocols is not listed separately here, but was similar to those who completed only one of the intervention diets. All data are reported as mean \pm SEM

MUFA monounsaturated fatty acids, PUFA polyunsaturated fatty acids

${ }^{1} p<0.017$ compared to CONT LFD.

$2_{p}<0.017$ compared to CONT HFD.

$3_{p}<0.017$ HFD versus LFD for the seven subjects who completed both diet interventions 


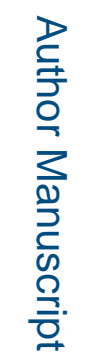

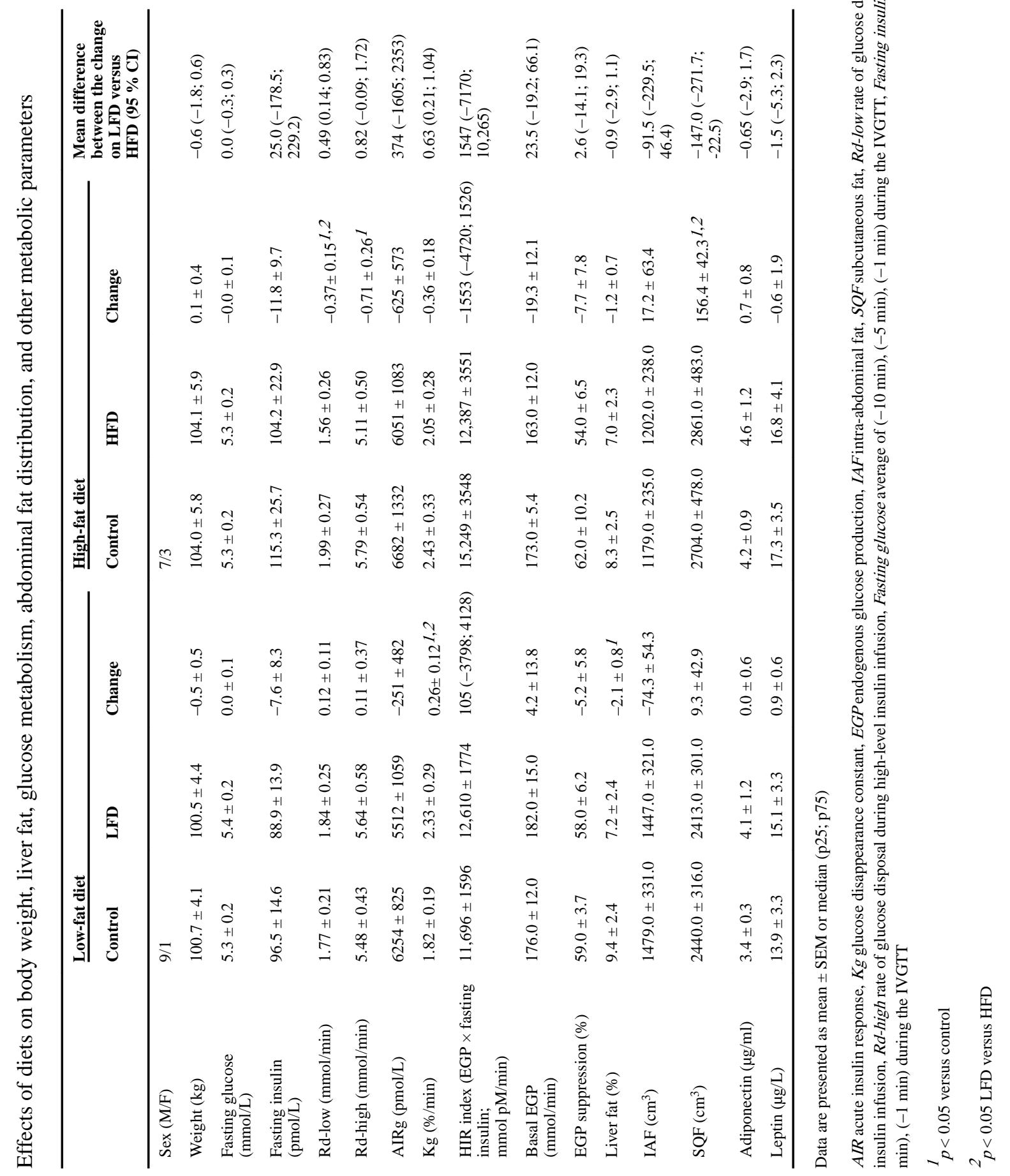

Eur J Nutr. Author manuscript; available in PMC 2018 February 01. 


\section{를

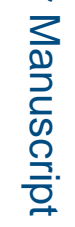

일

ב্

D

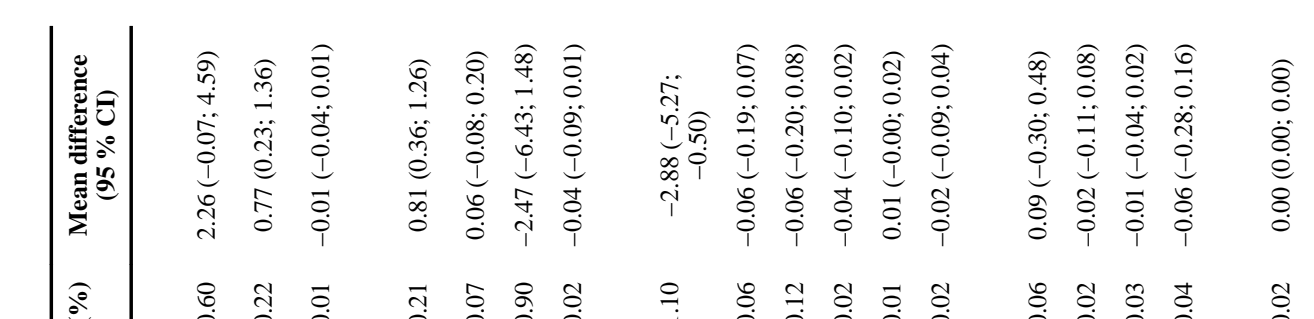

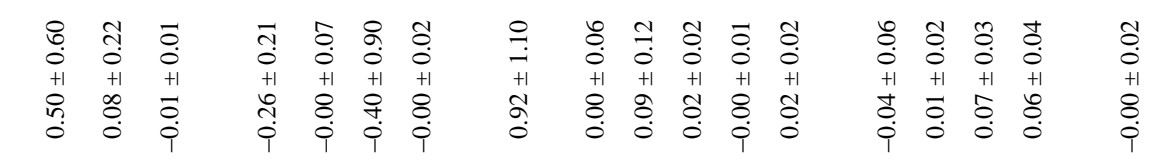

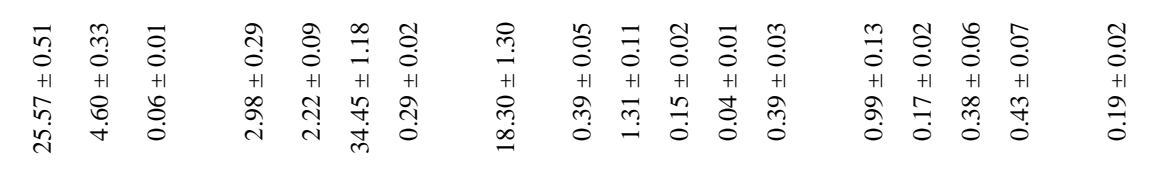

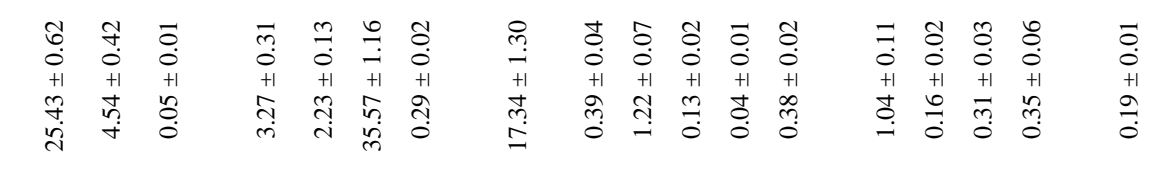

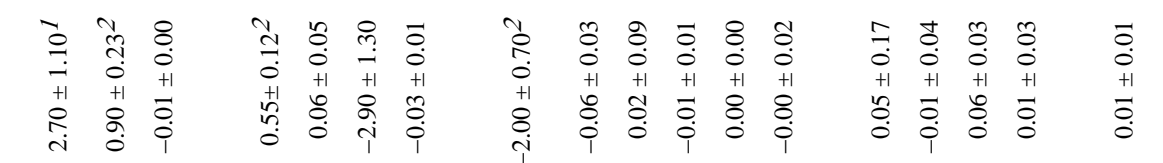

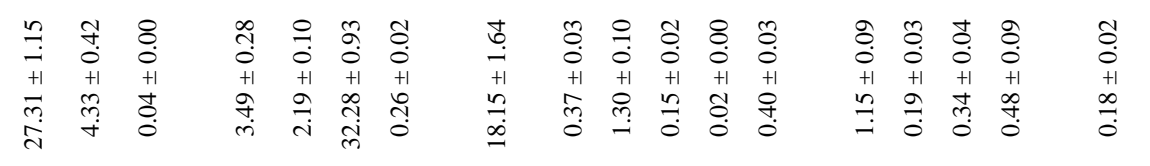

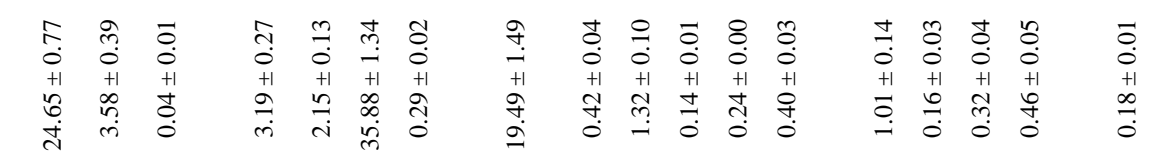

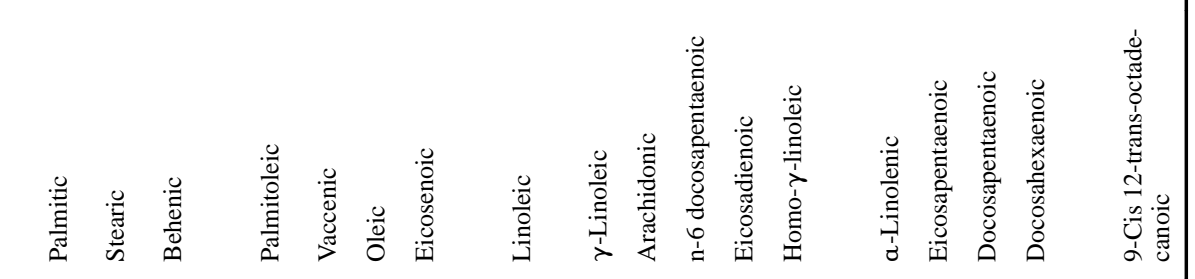

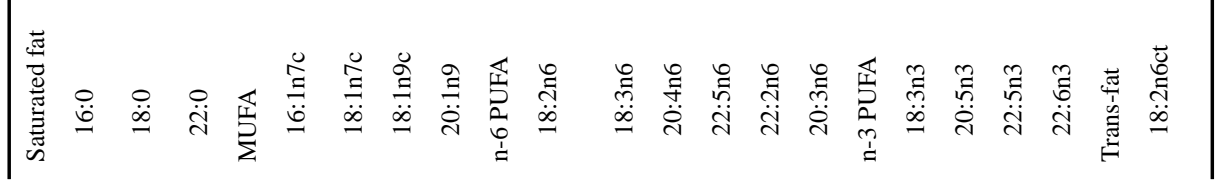

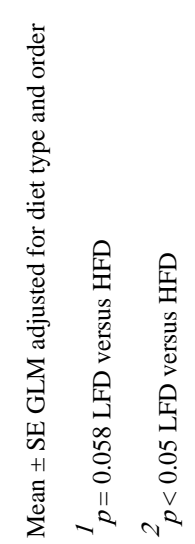

